\title{
A study of oral health in intestinal failure patients receiving home parenteral nutrition
}

\author{
A. M. Lee ${ }^{1}$, S. M. Gabe ${ }^{2}$, J. M. Nightingale ${ }^{3}$, J. Fiske $^{4}$ and M. Burke ${ }^{5}$ \\ ${ }^{1}$ Department of Oral and Maxillofacial Surgery, North West London Hospitals NHS Trust HA13UJ, ${ }^{2}$ Lennard-Jones \\ Intestinal Failure Unit, St Mark's Hospital HAl 3UJ, UK, Imperial College, ${ }^{3}$ Lennard-Jones Intestinal Failure Unit, \\ St Mark's Hospital HAl 3UJ, UK, ${ }^{4}$ Special Care Dentistry and ${ }^{5}$ Guys and St Thomas' NHS Foundation Trust, \\ London SE1 9RT, UK
}

There are no published oral health studies of people with long-term intestinal failure requiring intravenous nutrition (HPN). However, medical treatments used in this patient group can impact negatively on oral health and dental care delivery.

Fifty-two HPN outpatients were recruited from a specialised nutrition clinic of a National referral centre. Consenting patients listed their medical and medication history, perceived oral health and dental treatment in a structured interview and underwent an oral examination. Findings were compared with means from the 1998 UK Adult Dental Health Survey ${ }^{(1)}$ using one-sample $t$ tests. Additionally, an e-mail questionnaire was sent to the UK HPN Group, to determine views on oral health and prescribing practices of consultant gastroenterologists (68\% response rate).

The patient sample reflected the UK HPN population $\left(\mathrm{BAPEN}^{(2)}\right)$. Oral health of the HPN cohort was poorer than the UK norm; patients had more decay $(P<0.001)$ fewer teeth $(P<0.001)$ and fewer sound and untreated teeth $(P=0.023)$ despite similar dental attendance. Hyperphagia, sip feeds, oral rehydration fluids and polypharmacy (in $96 \%$ ), increase caries, xerostomia (in $81 \%$ ) and thus oral infection risk (including oral candidiasis). The patient pathway does not include oral health information. Management of HPN-related complications with bisphosphonate therapy, anticoagulation and parenteral antibiotic prophylaxis were associated with impaired access to dental care for $33 \%$. Patients were experiencing current problems $(60 \%)$ and psychological discomfort (56\%) from poor oral health, but were less anxious about dental treatment. Gastroenterologists report concern that dental disease/treatment could have an impact on patient health.

HPN patients have multiple risk factors that adversely affect oral health, and hence potential diet and well-being. Guidance on oral health and dental care provision is required.

1. Kelly M, Steele J, Nuttall N et al. (2000) Adult Dental Health Survey: Oral Health in the UK 1998. London: The Stationery Office.

2. BAPEN: British Association for Parenteral and Enteral Nutrition (2008) Annual BANS Report. Artificial Nutrition Support in the UK 2007 [B Jones B, editor]. Available at http://www.bapen.org.uk (accessed 07 April 2009). 